\title{
Mechanism for Electrostatic Repulsion or Attraction
}

\author{
Narahari V. Joshi \\ Department of Physics, University of Los Andes, Merida, Venezuela \\ Email: joshi@ula.ve
}

Received August 4, 2013; revised September 6, 2013; accepted September 29, 2013

Copyright (C) 2013 Narahari V. Joshi. This is an open access article distributed under the Creative Commons Attribution License, which permits unrestricted use, distribution, and reproduction in any medium, provided the original work is properly cited.

\begin{abstract}
It is known that there is a force of repulsion (or attraction) between two similar (or dissimilar) charges and the laws governing these forces are well established in electrostatics. However, the exact mechanism and the origin for these forces are not known. The purpose of this investigation, therefore, is to reveal these aspects in the light of the presence of vibrating strings and lines of fields created by the negative (or positive) charge. The present approach strongly suggests that the force of repulsion is originated between two charges due to fields synchronized with vibrating strings. The Gauss symmetry seems to play a crucial role in these aspects.
\end{abstract}

Keywords: Electrostatic Repulsion (or Attraction); Fluid Dynamics; Vibrating Strings; Microfluids

\section{Introduction}

Very recently, several important fundamental issues of electricity and magnetism have been addressed on the basis of vibrating strings [1]. In this investigation, all the aspects of string theory (e.g. dimensions) are not considered. It is only assumed that vacuum is filled with some vibrating elements known as strings; and according to Feynman's criteria [2], the situation is similar to a dry liquid (non viscous, compact, surface tension free). The presence of vacuum energy is confirmed by several experimental works including Casimir [3] effect and a considerable research has been made to convert vacuum energy into electromagnetic fields [4,5]. Every charged particle is associated with concentric circles of lines of fields (or lines of force) which are related with the electro-static energy. Electrostatic potential, force, energy, flux and other basic aspects of electric fields are discussed in several books [2].

A fresh view to these aspects has been provided according to which, concentric circles of lines of fields are associated with the organized collective motion of vibrating strings and converted into the force in the presence of a second charge [1]. Suppose that the charge is negative (e.g. electron), then the collective vibrations are in clock wise direction. According to the accepted principle of Gauss symmetry, if the particle has a positive charge, then the organized motion of the strings has to be in anti-clock wise direction. If charges are placed together, then the superposition of fields (or energy associ- ated with strings) takes place and an interesting situation emerges which helps to understand how and why the electrostatic force of repulsion (or attraction) is originated.

\section{Mechanism and Discussion}

Let us consider two electrons which are situated in the vacuum at points $\mathrm{A}$ and $\mathrm{B}$ together with the corresponding circles of organized vibrating strings correlated with lines of forces (Figure 1).

In fact, the spherical symmetry is broken by the presence of the second electron and the lines of fields should be altered according to the new symmetry. However, for simplicity let us consider that the spherical symmetry is not altered at the initiation and the charges are still not in motion and hence the magnetic field is absent. Let us examine the energy associated with the vibrating strings after the superposition of the field originated from the second electron. Strings are elements of dimensions of approximately $10^{-35}$ meter and are vibrating at a high frequency. It means that strings posses a considerable amount of energy in a small area. The exact magnitude is not easy to estimate as all relevant parameters are not known with precision. However, it is suggested that electron acts as a wake oscillator in the dry, compact liquid of strings and induces vibrations. Energy associated with them is inversely proportional to the distance from the charge particle. Other details are discussed earlier [1].

Now, let us analyze the stress originated in the strings due to the electrons located at the points A and B. For 


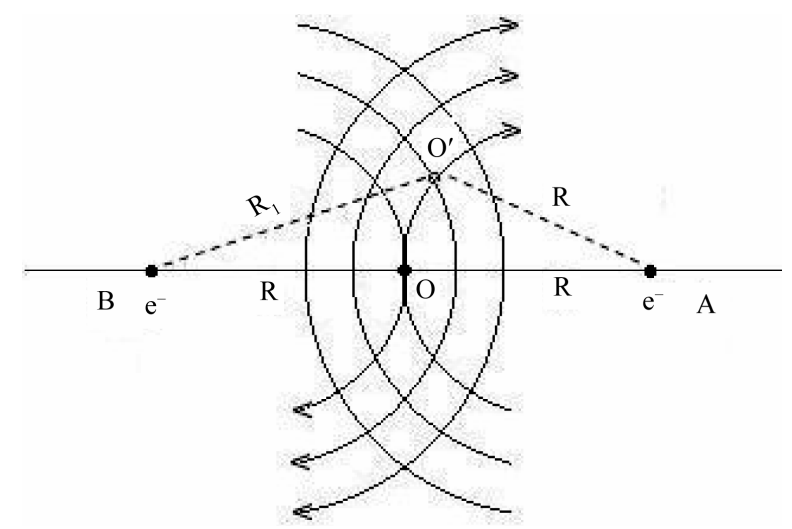

Figure 1. Electrons are located at the points $A$ and $B$ with corresponding lines of fields (clockwise direction).The stress is calculated at the mid point $O$ and at a typical point of intersection $O^{\prime}$ situated at the distances $R$ and $R_{1}$ from the point $A$ and $B$ respectively.

simplicity, let us consider the superposition at the midpoint $\mathrm{O}$. At this point, the stress is zero because the superposition of two lines of forces in opposite directions cancels the tension in the string. At the other points on the circle, the energy is also reduced with respect to the energy which it had before the presence of the second electron. Consider point $\mathrm{O}^{\prime}$, the intersection of two circles representing lines of forces. The difference in energy, $\mathrm{d} E$, originated from superposition between $\mathrm{O}$ and $\mathrm{O}^{\prime}$ is given by [1]

$$
\mathrm{d} E=J\left(\frac{1}{R}-\frac{1}{R_{1}}\right)
$$

where $J$ is a constant and $R$ and $R_{1}$ are distances from the electron located at $\mathrm{A}$ and $\mathrm{B}$. As $R_{1}$ is higher than $R$, the energy associated with strings in this space is higher as compared with the energy at the mid point O. Thus, a gradient of stress (or pressure), $\nabla P$, is created due to the superposition of tensions of the vibrating strings in the incompressible liquid towards the axis.

According to the fluid dynamics [6], the gradient of pressure $\nabla P$, (or tension) originates a fluid flow. In the present case, the net element of fluid is not conventional but made of strings in the "dry liquid" state. In this situation, the velocity of motion of the elements of the fluid (strings), $u$, is given by

$$
\begin{array}{ll}
\frac{\partial u}{\partial t}=0 & 0<t \\
\rho \frac{\partial u}{\partial t}=-\nabla P & 0<t>\mathrm{d} t \\
\frac{\partial u}{\partial t}=0 & t \geq \mathrm{d} t
\end{array}
$$

Here parameter $\rho$ is related with the density of strings and its motion towards (or away from) the axis separat- ing two charges. Equation (2) differs from the conventional equation which deals with the rate of flow of the liquid. In the present equation, important properties of fluid, namely the force of gravity, surface tension and viscosity details are absent. This is an unusual, extraordinary situation where the parameters of the moving element (the string) are not known. Equation (2) suggests that because of the pressure gradient, strings rush towards the axis joining two electrons; thus, separating them from each other. It is an instantaneous phenomenon as the change in the pressure or the tension in the strings takes place as soon as the second orbit overlaps the previous one. In Equation (2), the value of $\mathrm{d} t$, the time interval of motion of fluid, is not possible to estimate but it is expected to be extremely small till the balance of the pressure is established.

When the negative charge is replaced by the positive charge at the point $\mathrm{B}$, then lines of force of one superimpose on the other lines but in this case, the effect is different. Lines corresponding to electrons are clock wise meanwhile for a positive charge they are anti-close wise. So, tensions in the strings are added. This creates a higher pressure between two charge particles and the elements of the liquid, strings, rush away from the axis creating attraction between two opposite charge particles.

The situation is similar to the impulsive force of infinitesimal duration acting on the system. Differences in the pressure or in the tension created for a very short time by the superposition of lines of forces give rise to the movement of the elements (strings) of the fluid. In fact, the superposition of the concentric circles is not only limited to the region near the axis joining two charges but is spread in three dimensional space and obviously the strings acquire a flow motion from the higher pressure to the lower pressure in 3D space and create repulsion or attraction.

$U$ is the total potential energy of the vibrating strings and it is originated from the induced and organized vibrations of the strings due to the wake oscillator of the charge $\mathrm{q}$ of the electron [1]. As the existence of the electric field is associated with the vibrational energy of strings, which is proportional to the charge of the electron, $q$, the potential energy can be expressed as

$$
U=K q V
$$

where $K$ is a constant and $V$ is the potential energy associated with the electric field.

The gradient of potential energy is formed by the superposition of two circles of vibrating strings. As mentioned earlier, the points of intersections have less energy (in case of two similar charges) as the forces or stress energy is subtracted because they are in opposite directions due to the symmetry. Thus, the force is applied on the fluid by the fluid creating anisotropy in the space 
instead of spherical symmetry. Hence the gradient of energy is not only in the $X$ direction but also it has components in $Y$ and $Z$ directions. Obviously, the gradient of potential energy due to the superposition of lines of forces needs to be expressed in the tensor form and is given by

$$
F_{i}=-\sigma_{i j} \frac{\partial U}{\partial X j}
$$

As expected, Equation (4) is a tensor of second order.

$$
\begin{gathered}
F_{i}=\sigma_{i j} \frac{\partial K q V}{\partial X j} \\
F_{i}=K \sigma_{i j} q \frac{\partial V}{\partial X j}
\end{gathered}
$$

which can be written as

$$
F_{i}=K q \sigma_{i j} E_{j}
$$

Equation (7) is a familiar tensor form of the force on the charge $q$ due to the intensity of the electric field $E$.

The above analysis shows that the potential gradient in compact liquid plays a crucial role in the mechanics of the repulsion or attraction of two similar or dissimilar charges. First, the strings move towards the axis (in the case when both charges are similar) according to the potential gradient and help to separate them. Secondly, a force, which has a tensor character, is originated as a consequence of the variation of the potential energy in three dimensional space and helps to repel two similar charges.

This is very important and it has a different aspect. According to the conventional point of view, electrons repel each other. However, here we point out that the pressure associated with vibrational energy originated from strings (or the field) between two similar charges repels from the center of the two charges and hence the phenomenon of repulsion takes place. The role of the electromagnetic field in the repulsion (or attraction) process has been discussed earlier in electrodynamics [7] and the present approach provides a strong basis for it. The only difference is that, here, the role of the magnetic field is not considered as the charge particles still are not in movement.

The present view differs from the conventional one as it considers that the electrostatic repulsion (or attraction) is originated not at the electron but between two charges and the source lies in the energy associated with the vi- brating strings. The electron synchronies and organizes the motion of the strings and makes available the energy according to the symmetry of the field which is used to repel or attract the same or the opposite charge. This is the first time that the exact mechanism for electrostatic forces is provided. This will help to understand basic phenomena in physics.

\section{Conclusion}

The main purpose of the present approach is to explain the basic issues raised by Feynman, namely "what flows with the flux of the electric field and what is happening between two charges which makes them repel or attract". This is achieved by considering the presence of vibrating strings in the form of a dry, compact and incompressible fluid. The presence of a second charge originates superposition of two circles which represent lines of forces (or fields) and causes a pressure gradient in the compressed liquid. It has double consequences. The first is that the strings flow towards the axis separating two similar charges and secondly it originates a force tensor as shown in Equation (7). A direct experimental confirmation may be obtained by measuring forces precisely in geometries of different symmetries for a set of charges.

\section{REFERENCES}

[1] N. V. Joshi, "Energy Conversion from the Vacuum Field to Electromagnetic Fields," Physics Essays, Vol. 26, No. 1, 2013, pp. 61-67. http://dx.doi.org/10.4006/0836-1398-26.1.61

[2] R. P. Feynman, R. B. Leighton and M. Sands, "The Feynman Lectures on Physics Vol. 2," Addition Wesley, Boston, 1969.

[3] R. L. Jaffe, "Casimir Effect and the Quantum Vacuum," Physical Review D, Vol. 72, No. 2, 2005, Article ID: 021301(R). http://dx.doi.org/10.1103/PhysRevD.72.021301

[4] B. Haisch and G. Moddel, "Quantum Vacuum Energy Extraction," US Patent No. 7379286, 2008.

[5] T. E. Bearden, "Energy from the Vacuum, Chapters 7 and 2," Cheniere Press, UK, 2004.

[6] C. K. Batchelor, "An Introduction to Fluid Dynamics, Chapters 5 and 6," Cambridge University Press, Cambridge, 1993.

[7] W. T. Grandy, "Introduction to Electrodynamic," Academic Press, New York, 1970. 